\title{
Atividade inseticida do óleo essencial de Tanaecium nocturnum (Barb. Rodr.) Bur. \& K. Shum (Bignoneaceae) sobre Sitophilus zeamais Motsch. (Coleoptera: Curculionidae)
}

\author{
Murilo FAZOLIN ${ }^{1}$, Joelma Lima Vidal ESTRELA², Valdomiro CATANI ${ }^{3}$, Márcio Rodrigo ALÉCIO ${ }^{4}$, Marilene \\ Santos de LIMA² \\ RESUMO \\ O óleo essencial extraído de folhas frescas de Tanaecium nocturnum (Barb. Rodr.) Bur.\& K. Shum por destilação de arraste a \\ vapor foi avaliado quanto à toxicidade a Sitophilus zeamais Motsch., principal praga do milho armazenado. Papel de filtro e grãos \\ de milho foram impregnados pelo óleo para se avaliar o efeito por via de contato (papel-filtro) e fumigação, respectivamente. \\ Para avaliação do efeito da aplicação tópica $0,5 \mu \mathrm{l}$ das diferentes concentraçôes do óleo foram aplicadas em adultos do inseto. \\ A partir de uma ampla faixa de concentraçôes, foram determinadas as mais promissoras para os bioensaios definitivos. $\mathrm{Na}$ \\ determinação das dose/concentrações-letais $\left(\mathrm{DL}_{50}\right.$ e $\left.\mathrm{CL}_{50}\right)$ foi utilizada a análise de Probit, realizando-se também, uma análise \\ de regressão linear conjunta de todos os dados de mortalidade. O óleo de T. nocturnum foi considerado tóxico para S. zeamais \\ baseado nos seguintes valores: $\mathrm{CL}_{50}$ de 14,1 ng. $\mathrm{cm}^{-2} \mathrm{e} \mathrm{CL}_{50}$ de 1.321,6 ng. $\mathrm{g}^{-1}$ de grãos para os efeitos de contacto (papel-filtro) \\ e fumigação, respectivamente, e $\mathrm{DL}_{50}$ de $14,7 \mu \mathrm{g} . \mathrm{mg}^{-1}$ de inseto para efeito tópico. Porcentagens de mortalidade próximas \\ a $100 \%$ foram obtidas nas concentrações de: 2 e $5 \%$ (m/v) (contato), 3 4, e $5 \%(\mathrm{~m} / \mathrm{v})$ (fumigação) e $10 \%(\mathrm{~m} / \mathrm{v})$ para o \\ efeito de aplicação tópica. O presente estudo mostrou que o ácido cianídrico, liberado do óleo essencial de T. nocturnum por \\ hidrólise, pode ter atividade inseticida para $S$. zeamais e que concentrações acima de $4 \%(\mathrm{~m} / \mathrm{v})$ são promissoras no controle \\ do inseto.
}

PALAVRAS-CHAVE: Kangàrà kanê, Cipó vick, Inseticida botânico, Mandelonitrila, Toxicidade.

\section{Insecticide activity of the essencial oil Tanaecium nocturnum (Barb. Rodr.) Bur. \& K. Shum (Bignoneaceae) on Sitophilus zeamais Motsch. (Coleoptera: Curculionidae)}

\begin{abstract}
Insecticidal activities of Tanaecium nocturnum (Barb. Rodr.) Bur. \& K. Shum (Bignoneaceae) essential oil against Sitophilus zeamais Motsch. (Coleoptera: Curculionidae). The essential oil extracted from fresh leaves of the Tanaecium nocturnum (Barb. Rodr.) Bur.\& K. Shum by steam distillation, was tested against maize weevil Sitophilus zeamais Motsch.. Filter paper and maize grains impregnation was employed, for contact and fumigant toxicity effect studies, respectively. For topical effect studies, aliquots of $0,5 \mu \mathrm{l}$ of the diluted oil applied to adults insects. We used a broad range of concentrations to be used for establish the most promising concentrations. Probit analysis was employed in analyzing the dose/ concentration-mortality response $\left(\mathrm{LD}_{50}\right.$ and $\left.\mathrm{LC}_{50}\right)$. Linear regression analysis was applied in mortality of maize weevil adults. The toxicity $\left(\mathrm{LC}_{50}\right)$ T. nocturnum oil to $S$. zeamais was of $14.1 \mathrm{ng} . \mathrm{cm}^{-2}$ and $\mathrm{LC}_{50}$ of $1,321.6 \mathrm{ng} \cdot \mathrm{g}^{-1}$ of the grains for contact and fumigant effects, respectively, and $\mathrm{LD}_{50}$ of $14.7 \mu \mathrm{g} \cdot \mathrm{mg}^{-1}$ of the insect for topical application. For contact and fumigant effects, the mortality rate was nearly $100 \%$ at oil concentrations of $2 \%$ and $5 \%(\mathrm{~m} / \mathrm{v})$, and $4 \%$ and $5 \%(\mathrm{~m} / \mathrm{v})$, respectively. For contact, fumigant, and topical effects, the mortality rate was nearly $100 \%$ at oil concentrations of $2 \%$ and $5 \%(\mathrm{~m} / \mathrm{v}) ; 4 \%$ and $5 \%(\mathrm{~m} / \mathrm{v})$ and $10 \%(\mathrm{~m} / \mathrm{v})$ respectively. The current study shows that cyanidric acid, liberated from the essential oil of the T. nocturnum, posses fumigant insecticidal effect on S. zeamais in concentrations more than $4 \%(\mathrm{~m} / \mathrm{v})$ were promises.
\end{abstract}

KEY-WORDS: Kangàrà kanê, Cipó vick, Botanical insecticide, Mandelonitrile, Toxicity.

1 Embrapa Acre. Caixa Postal 321, 69908-970, Rio Branco, Acre. e-mail: murilo@cpafac.embrapa.br

2 Embrapa Acre/CNPq. e-mail: joelma@cpafac.embrapa.br

3 Embrapa Acre. e-mail: catani@cpafac.embrapa.br

${ }^{4}$ e-mail: mralecio@yahoo.com.br 


\section{INTRODUÇÃO}

Plantas do gênero Tanaecium encontram-se distribuídas nas Américas Central e do Sul e no Oeste da Índia, sendo relatado um total de dezenove espécies (Schultes \& Raffauf, 1995).

O cipó de "kangàrà kanê" Tanaecium nocturnum (Barb. Rodr.) Bur \& K. Shum. (Bignoniaceae) foi relatado como inseticida por Kerr \& Posey (1991), baseando-se em sua utilização pelos índios Kayapós (Pará) para matar abelhas.

Outras formas de utilização dessa planta dependem da etnia indígena considerada, sendo usada como antidiarréica pela tribo Karitiana (Rondônia) e alucinógena pelos Paumari (Amazonas) (Prance et al., 1977). O poder afrodisíaco da planta é apontado pela tribo Chocó (Colômbia) (Gentry, 1973).

O óleo essencial do caule de T. nocturnum possui em sua composição 96 \% de benzaldeído (Gottlieb et al., 1981). Esta substância é utilizada no preparo de certos pigmentos de anilina, e de outros produtos como perfumes, flavorizantes e solventes, sendo que em altas concentraçōes é considerado narcótico podendo causar dermatite por contato (Budavari et al., 1985). Folhas de louro (Laurus nobilis L.) contêm compostos benzaldeído, cuja propriedade repelente a insetospraga de grãos armazenados foi comprovada para Rhyzoperta dominica (Fabr.), Sitophilus oryzae (L.) e Tribolium castaneum (Herbst) (Jillani \& Saxena, 1990; Xie et al., 1995).

T. nocturnum é abundante nas florestas do Estado do Acre, necessitando de uma avaliação sobre sua utilização como inseticida botânico, principalmente durante o armazenamento de grãos por pequenos produtores familiares. Neste contexto, dentre as pragas que infestam grãos armazenados no Brasil, o gorgulho Sitophilus zeamais Mots., 1855 (Coleoptera: Curculionidae) é o mais importante, devido ao grande número de hospedeiros, elevado potencial biótico, capacidade de penetração na massa de grãos e infestação cruzada, ocasionando danos principalmente, aos grãos de milho, arroz e trigo (Gallo et al., 2002). O controle desses insetos tem sido comumente, realizado em larga escala por meio de produtos químicos, muitas vezes aplicados de forma inadequada. As pesquisas atuais e o aumento no conhecimento dos efeitos indesejáveis advindos do uso indiscriminado desses produtos associados à preocupação dos consumidores quanto à qualidade de alimentos, tem incentivado estudos relacionados a novas técnicas de controle dessas pragas (Tavares, 2002).

O objetivo deste trabalho foi avaliar a toxicidade do óleo essencial de T. nocturnum para adultos de S. zeamais.

\section{MATERIAL E MÉTODOS}

\section{COLETA DAS PLANTAS, PROCESSAMENTO E EXTRAÇÃO DO ÓLEO ESSENCIAL}

As plantas utilizadas na extração do óleo foram coletadas na área florestal da Embrapa Acre, localizada na Rodovia BR 364 no quilômetro 14 sentido Rio Branco-Porto Velho, no município de Rio Branco-AC. Foram selecionadas plantas que apresentaram secções de caule de até $1 \mathrm{~cm}$ de diâmetro, que no laboratório foram submetidas a uma separação entre folhas e ramos e imediatamente submetidas à extração por arraste de vapor de água, utilizando-se o um destilador do tipo Clevenger. O rendimento foi de $0,8 \%$ de óleo essencial obtido das folhas e de $0,7 \%$ dos talos.

A análise do óleo essencial de T. nocturnum foi realizada em cromatógrafo gasoso (VARIAN, modelo 3800) acoplado a espectrômetro de massas (VARIAN-SATURN 2000). Os resultados apontaram como componente principal o benzaldeído (88,4\%), concordando com o trabalho precedente de Gottlieb et al. (1981). Por meio da análise qualitativa do óleo essencial por cromatografia gasosa acoplada ao detector de massas, utilizando-se a técnica de injeção direta via CromatoProb, foi possível identificar o benzaldeído, $\alpha$-(benziloxi)-mandelonitrila e mandelonitrila (Figura 1). Foi determinada uma concentração média de $636 \mathrm{ppm}$ de ácido cianídrico nas folhas recém-coletadas de T. nocturnum, sugerindo que seja este o provável responsável pelo princípio inseticida do óleo.

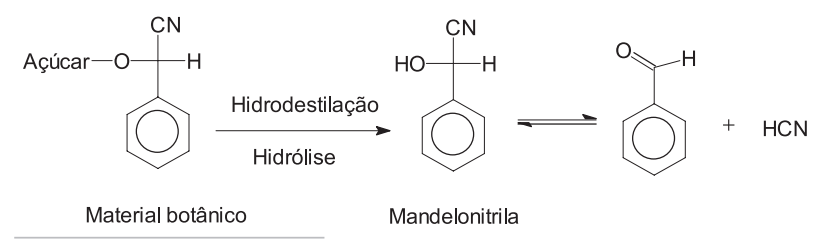

Figura 1- Esquema da hidrólise da mandelonitrila.

\section{BIOENSAIOS PRELIMINARES}

Os experimentos foram conduzidos no Laboratório de Entomologia da Embrapa Acre, localizada na Rodovia BR 364 no quilômetro 14 sentido Rio Branco-Porto Velho, no município de Rio Branco-Acre, sendo utilizados adultos de Sitophilus zeamais obtidos de uma criação natural em sacos de milho previamente infestados pela praga. Os indivíduos utilizados nas avaliações pesaram em média $3,1 \mathrm{mg}$, sendo que nos bioensaios para avaliação dos efeitos de contato e aplicação tópica, foram utilizadas placas de Petri medindo $9,0 \mathrm{~cm}$ de diâmetro por $1,5 \mathrm{~cm}$ de altura. Para avaliação do efeito por fumigação foram utilizados potes plásticos com capacidade 
para $250 \mathrm{ml}$, fechados com tampa hermética. Em todos os casos os recipientes foram mantidos em câmara climatizada à temperatura de $25 \pm 2{ }^{\circ} \mathrm{C}$, umidade relativa de $70 \pm 5 \% \mathrm{e}$ fotofase de $12 \mathrm{~h}$.

\section{DETERMINAÇÃO DAS CURVAS DE CONCENTRAÇÃO- MORTALIDADE E EFICIÊNCIA DO ÓLEO DE T. nocturnum}

Testes preliminares para avaliação da intoxicação dos insetos por exposição de contato em superfície contaminada (papel-filtro), aplicação tópica e fumigação foram realizados visando à obtenção de intervalos de concentração do óleo compreendidos entre a concentração que não ocasionasse mortalidade dos insetos e a que proporcionasse mortalidade de todos os indivíduos submetidos à avaliação. Cada faixa de resposta foi obtida a partir do óleo puro, na forma líquida, submetido às diluições seqüenciais em acetona, obtendo-se as concentrações em unidades de massa de óleo em volume de acetona $(\mathrm{m} / \mathrm{v})$. Foi realizado concomitantemente, um teste de sobrevivência de adultos de $S$. zeamais submetidos à exposição à acetona em todas as vias de contaminação avaliadas. Desta forma, dentro do intervalo de concentraçóes testado nos bioensaios iniciais, foram determinadas faixas mais estreitas para avaliação nos bioensaios definitivos.

A faixa de resposta obtida para o efeito de contato (papelfiltro) foi de $0,01 \%(\mathrm{~m} / \mathrm{v})$ a $5,0 \%(\mathrm{~m} / \mathrm{v})$, para aplicação tópica de $3,0 \%(\mathrm{~m} / \mathrm{v})$ a $10,0 \%(\mathrm{~m} / \mathrm{v})$, fumigação de $0,1 \%(\mathrm{~m} / \mathrm{v})$ a $5,0 \%(\mathrm{~m} / \mathrm{v})$. Dentro destes intervalos foram utilizadas entre cinco e seis concentraçōes para os bioensaios definitivos, além de um controle com apenas solvente. Essas concentrações foram utilizadas para determinação das respectivas curvas de concentração-mortalidade e cálculo da concentração/dose com probabilidade de causar $50 \%$ de mortalidade $\left(\mathrm{CL}_{50} \mathrm{e}\right.$ $\mathrm{DL}_{50}$ ) dos insetos submetidos à experimentação, seguindo metodologia descrita por Finney (1971).

Foram realizados ainda testes de ajuste com o propósito de determinar os padrões ideais dos seguintes fatores: tempo de exposição dos insetos ao óleo, volume do produto a ser utilizado e o número de insetos por parcela experimental.

\section{BIOENSAIOS DEFINITIVOS}

Determinação do efeito de contato em superfície contaminada (papel-filtro)

Os bioensaios de concentração-mortalidade foram realizados impregnando-se papeis-filtro com $0,5 \mathrm{ml}$ das seguintes concentraçôes do óleo de T. nocturnum: 0,01; 0,1; 1,$0 ; 2$ e $5 \%(\mathrm{~m} / \mathrm{v})$. Após evaporação do solvente, em média por 10 minutos, os papeis-filtro foram colocados nas placas de Petri, que em seguida receberam 20 adultos de S. zeamais/ placa. Como testemunha, outro grupo de 20 indivíduos foram colocados em placa de Petri contendo papel-filtro impregnado com $0,5 \mathrm{ml}$ de acetona pura.
O tempo de exposição aos tratamentos foi de $48 \mathrm{~h}$ para posterior avaliação da mortalidade dos insetos.

\section{DETERMINAÇÃO DO EFEITO POR APLICAÇÃO TÓPICA}

Os adultos de $S$. zeamais foram anestesiados por congelamento a $-16^{\circ} \mathrm{C}$, em freezer doméstico, durante dois minutos, suficiente apenas para que fossem paralisados para manipulação e colocados em placas de Petri em grupos de 10 indivíduos. Cada inseto recebeu $0,5 \mu \mathrm{l}$ de óleo essencial no pronoto, aplicado por meio de um microsseringa de $10 \mu \mathrm{l} \mathrm{de}$ capacidade, nas seguintes concentraçôes em acetona: 3,0; 4,0; 5,$0 ; 6,0 ; 8,0$ e $10,0 \%(\mathrm{~m} / \mathrm{v})$. Outro grupo de 10 indivíduos recebeu no pronoto $0,5 \mu$ de acetona pura.

O tempo de exposição aos tratamentos foi de $48 \mathrm{~h}$ para posterior avaliação da mortalidade dos insetos.

\section{DETERMINAÇÃO DO EFEITO POR FUMIGAÇÃO}

Os bioensaios foram instalados utilizando-se de grãos de milho para cada pote plástico de $250 \mathrm{ml}$. Nesta massa de grãos foi aplicado $2 \mathrm{ml}$ de solução de óleo em acetona nas concentraçôes de 0,$1 ; 1,0 ; 2,0 ; 3,0 ; 4,0 ;$ e 5,0 \% (m/v). Após o revolvimento para homogeneização, deixou-se secar por 20 minutos para volatilização do solvente, para em seguida confinar em cada frasco, 20 adultos de $S$. zeamais. Como testemunha, outro grupo de 20 indivíduos foram colocados na mesma massa de grãos impregnados com $2 \mathrm{ml}$ de acetona pura. Novamente avaliou-se a mortalidade dos insetos, após exposição aos tratamentos por $48 \mathrm{~h}$.

\section{ANÁLISE DOS DADOS}

Para todos os bioensaios, os valores de mortalidade dos insetos submetidos aos tratamentos foram corrigidos pela fórmula de Abbott (1925), levando-se em consideração a mortalidade ocorrida nos tratamentos testemunha. Os dados de mortalidade das concentraçóes-resposta, assim corrigidos, foram submetidos à análise de Probit, utilizando-se o programa de análises estatísticas SAS (SAS Institute 1997). Depois de estimadas as $\mathrm{DL}_{50}$ para o efeito da aplicação tópica e $\mathrm{CL}_{50}$ para as demais avaliaçōes, estas foram usadas como concentraçōes discriminatórias na detecção da toxicidade em relação a $S$. zeamais.

O delineamento utilizado foi inteiramente ao acaso, com quatro repetiçôes, sendo cada bioensaio repetido três vezes, realizando-se uma análise de regressão linear conjunta de todos os dados de cada via de exposição, pelo procedimento SAS (SAS Institute, 1997). 


\section{RESULTADOS E DISCUSSÃO}

$\mathrm{O}$ valor da $\mathrm{CL}_{50}$ de $1,41 \mathrm{ng}$ de óleo.cm² ${ }^{-2}$, estimado para o óleo de T. nocturnum na exposição por contato (papel-filtro) (Tabela 1) foi inferior aos obtidos por Ribeiro et al. (2003), para populaçōes de $S$. zeamais susceptíveis aos inseticidas piretróides sintéticos a base de deltametrina, cipermetrina, e permetrina $\left(80 ; 115 ; 490 \mathrm{ng}\right.$ de princípio ativo. $\mathrm{cm}^{-2}$, respectivamente) e ao organofosforado malathion $(31 \mathrm{ng}$ de óleo. $\left.\mathrm{cm}^{-2}\right)$. A mesma tendência foi observada comparandose estes valores com os obtidos por Huang et al. (1998) em avaliaçôes de toxicidade a este inseto exposto ao óleo essencial de Myristica fragans Houtt (1.740 ng de óleo. $\mathrm{cm}^{-2}$ ).

Para a exposição por via tópica, o valor estimado da $\mathrm{DL}_{50}$ do óleo essencial de T. nocturnum (14,7 ng. $\mathrm{mg}^{-1}$ de inseto) para S. zeamais foi 3.537 vezes inferior ao estimado por Huang et al. (2000) utilizando o óleo de Ellateria cardamomum (L.) Maton.

A curva de concentração-mortalidade do óleo de $T$. nocturnum apresentou maior inclinação para a exposição por fumigação (Tabela 1), quando comparado aos demais modos de exposição, demonstrando que adultos de $S$. zeamais respondem de forma mais homogênea a aplicação do óleo por esta via.

As vias de intoxicação por contato (papel-filtro) e fumigação foram mais eficazes no controle do inseto uma vez que porcentagens de mortalidade próximas a $100 \%$ foram obtidas em concentraçōes de 2,0 e 5,0 \% (m/v) para contato (papel-filtro) e 4,0 e 5,0 \% (m/v) para fumigação (Figura 2).

A variância explicada pela relação da mortalidade de $S$. zeamais em função da concentração do óleo de $T$. nocturnum (coeficiente de determinação) foi de $0,71 \mathrm{e}$ 0,85 para os efeitos de contato (papel-filtro) e fumigação, respectivamente, reforçando a linearidade do efeito por
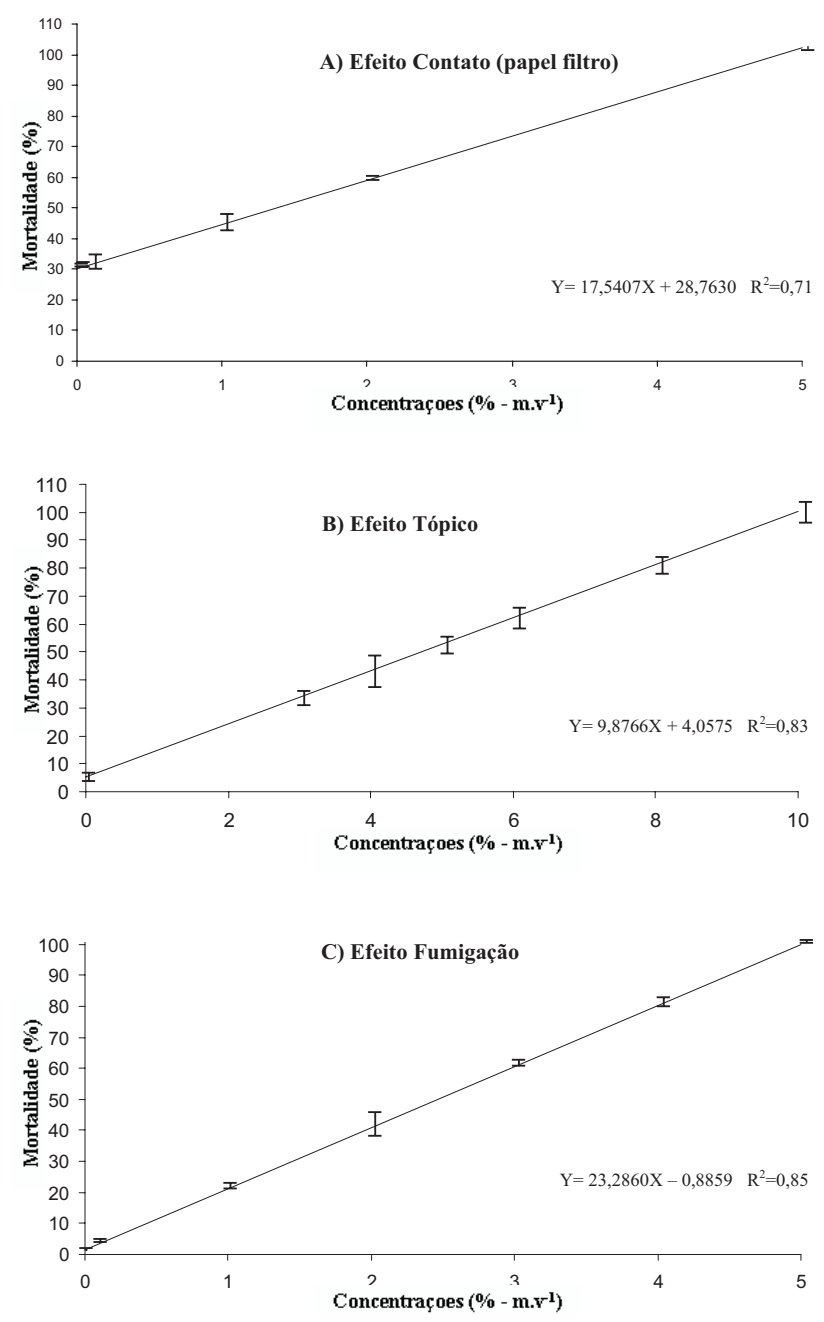

Figura 2 - Curva de mortalidade de Sitophilus zeamais Motsch. em função das diferentes concentrações e vias de exposição ao óleo essencial de Tanaecium nocturnum (Barb. Rodr.) Bur. \& K. Shum.

Tabela 1 - Toxicidade do óleo essencial de Tanaecium nocturnum (Barb. Rodr.) Bur. \& K. Shum para adultos de Sitophilus zeamais Motsch.

\begin{tabular}{|c|c|c|c|c|c|c|}
\hline Vias de exposição & $\mathrm{n}$ & Inclinação \pm EPM & $\mathrm{CL}_{50}(\mathrm{IC} 95 \%)$ & $\mathrm{DL}_{50}(\mathrm{IC} 95 \%)$ & $\chi^{2}$ & Prob. \\
\hline Contato (papel-filtro) & 480 & $0,4580 \pm 0,0313$ & $\begin{array}{l}14,1(10,8-17,9) \\
\text { ng de óleo/(cm² }\end{array}$ & - & 3,1055 & 0,2117 \\
\hline Fumigação & 560 & $1,9790 \pm 0,1543$ & $\begin{array}{l}\text { 1.128,3 }(1.028,1-1.238,5) \\
\text { ng de óleo/g de grãos }\end{array}$ & - & 34,9302 & 0,0100 \\
\hline $\begin{array}{l}\text { Aplicação } \\
\text { Tópica }\end{array}$ & 280 & $1,2789 \pm 0,1634$ & - & $\begin{array}{l}14,7(13,7-15,6) \\
\text { ng de óleo/mg de inseto }\end{array}$ & 7,3764 & 0,1173 \\
\hline
\end{tabular}

$\mathrm{n}=$ número de insetos submetidos ao ensaio, $\mathrm{EPM}=$ Erro padrão da média, $\mathrm{CL}_{50}$ e $\mathrm{DL}_{50}=$ Concentração e Dose Letal, respectivamente, que causam $50 \%$ de mortalidade e IC $95 \%=$ Intervalo de Confiança a $95 \%$ de probabilidade, 
fumigação. Provavelmente a mortalidade do inseto por fumigação poderá estar relacionada ao ácido cianídrico produzido pela hidrólise da mandelonitrila (Figura 1).

Para a via de exposição tópica, porcentagens de mortalidade acima de $80 \%$ foram obtidas somente em concentraçóes mais altas, ou seja, 8,0 e $10,0 \%(\mathrm{~m} / \mathrm{v})$ respectivamente, apresentando um coeficiente de determinação de 0,83 (Figura 2B).

O óleo essencial de T. nocturnum apresenta efeito inseticida para $S$. zeamais, sendo que na sua utilização no controle do inseto concentraçôes acima de $4 \%$ podem ser consideradas promissoras.

\section{AGRADECIMENTOS}

Os autores agradecem o financiamento da pesquisa por parte do Conselho Nacional de Desenvolvimento Científico e Tecnológico (CNPq) e o apoio técnico de Valdemir de Souza e Silva, Ronaldo Queiroz de Oliveira, Charles Rodrigues da Costa, Rubens Mamedio Bastos, John Lennon Mesquita Catão e Pedro Pereira da Silva, todos funcionários da Embrapa Acre.

\section{BIBLIOGRAFIA CITADA}

Abbott, W.S. 1925. A method of computing the effectiveness of an insecticide. J. Econ. Emtomol. 18: 265-267.

Budavari, S.M., O'Neil, J., Smith, A., Hecleman, P.E.1985. The Merck index: an encyclopedia of chemical, drugs and biologicals. Merck Company, Incorporation, New Jersey, USA. 324pp.

Finney, D.J. 1971. Probit analysis. Cambridge University Press, London. 33 pp.

Gentry, A. 1973. Flora of Panama. Family 172. Bignoniaceae. Ann. Mo. Bot. Gdn., 60: 957.

Gallo, D., Nakano, O., Silveira Neto, S., Carvalho R.P.L., Batista, G.C., Berti Filho, E., Parra, J.R.P., Zucchi, R.A., Alves, S.B., Vendramim, J.D., Marchini, L.C., Lopes, J.R.S., Omoto, C. 2002. Entomologia Agrícola. Piracicaba: FEALQ. 920pp.

Gottlieb, O.R.; Koketsu, M.K.; Magalhães, M.T.; Maia, J.G.S.; Mendes, P.H.; Rocha, A.I.; Silva, M.L.; Wilberg, V.C. 1981. Óleos essenciais da Amazônia., VII. Acta Amazônica, 11 (1): 143-148.
Huang, Y.; Ho, S.H. 1998. Toxicity and antifeedant activities of Cinnamaldehyde against the grains storage insects, Tribolium castaneum (Herbst) and Sitophilus zeamais Motsch. Jour. Stored. Prod. Res., 34: 11-17.

Huang, Y.; Lam, S.L.; Ho, S.H. 2000. Bioactivities of essential oil from Ellataria cardamomum (L.) to Sitophilus zeamais Motschulsky and Tribolium castaneum (Herbst). Jour. Stored. Prod. Res., 36: 107-117.

Jillani, G. \& Saxena, R.C. 1990. Repellent and feeding deterrent effects of turmeric oil, sweetflay, neem oil, and neem leared insecticida against lesser grain borer (Coleoptera: Bostrichidae). J. Econ. Entomol., 83: 629-634.

Kerr, W.E.; Posey, D. A. 1991. "Kangàrà Kanê" Tanaecium nocturnum (Bignoniaceae), um cipó usado pelos índios Kayapós como inseticida natural. Acta Amazônica, 7(1): 23-26.

Prance, G.T.; Campbell, D.G.; Nelson, B.W. 1977. The etnobotany of Paumari indians. Econ. Bot., 31: 119-175.

Ribeiro, B.M.; Guedes, R.N.C.; Oliveira, E.E.; Santos, J.P. 2003. Insecticide resistance and synergism Brasilian populations of Sitophilus zeamais (Coleoptera: Curculionidae). Jour. Stored. Prod. Res., 39: 21-31.

SAS Institute. 1977. SAS User's Guide: statistics. Version 6.12. SAS Institute, Cary, NC, USA.

Schultes, R. E.; Raffauf, R.F. 1995. The healing forest. Dioscorides Press, Oregon, USA. 484pp.

Tavares, M.A.G.C. 2002. Bioatividade da erva-de-santa-maria, Chenopodium ambrosoides $L$ (Chenopodiaceae), em relação a Sitophilus zeamais Mots., 1855 (Col: Curculionidae). Dissertação de Mestrado, Escola Superior de Agricultura Luiz de Queiroz, Universidade de São Paulo, Piracicaba, São Paulo, SP. 59pp.

Xie, Y.S., Fields, P.G., Isman, M.B. 1995. Repellency and toxicity of azadirachthin and neem concentrates of three stored-product insects. J. Econ. Entomol., 88: 1024-1031.

Recebido em 25/11/2004

Aceito em 04/09/2007 
